\title{
Main Producers of World Cancer Epidemic
}

\author{
Robert Skopec ${ }^{*}$ \\ Researcher-analyst, Dubnik, Slovakia
}

*Corresponding author: Robert Skopec, Researcher-analyst, Dubnik, Slovakia, E-mail: zxcbnvm7@gmail.com

\begin{abstract}
There is a growing amount of the research data confirming the cancer risk associated with cooking starchy foods at high temperatures. In a word, acrylamide is a chemical used in lots of industrial processes, including water purification, or to separation of DNA molecules in experiments. Animal studies clearly show that acrylamide causes all sorts of cancers. There's no reason to think that it wouldn't produce cancer similarly in humans too. Acrylamide might be responsible for a long lasting and not enough known World Epidemic of Cancer. A growing number of studies are finding evidence that pesticides used in home gardening and agriculture are also associated with increased cancer risk. Glyphosate-based herbicides are leading products of the current pesticide market, and this trend has further strengthened outside Europe with the spread of glyphosate-tolerant GM crops. Some compounds find their way into the food supply through agricultural use, animal farming, or food processing, even if their use is not directly intended for human consumption. Concern about the possible effects of food additives on health, including cancer, is one reason that many people are now interested in organic foods.
\end{abstract}

\section{Keywords}

Acrylamide, Cooked foods, Processed meat, Red meat, Carcinogenesis, Protection against World Epidemic of Cancer

\section{Introduction}

Acrylamide is also found in several foods, and is made by the Maillard reaction, which browns cooked foods (gives them their pleasing flavour). While sugars and amino acids react together, they produce many of different chemicals. Particularly high levels of acrylamide are found in starchy foods, like potatoes and bread, when cooked at temperatures over $120^{\circ} \mathrm{C}$. This chemical can be also present in breakfast cereals, biscuits, coffee. In the organism, acrylamide is converted into another compound, glycidamide, which can bind to DNA and cause mutations.
The more acrylamide you consume, the higher the risk is likely to be. The advice "go for gold" aims for a golden yellow color or lighter when frying, baking, and toasting or roasting starchy foods is not enough. You might do well to eat them less often and amount. These kind of trade-offs are also representing a universal adequate prevention method. The occasional meal that's a bit overcooked can be also dangerous. This is about managing risk across whole lifetime. For example, don't keep raw potatoes in the fridge. At low temperatures, an enzyme called invertase breaks down the sugar sucrose into glucose and fructose, which can form acrylamide during cooking. Frozen food doesn't carry this particular risk, as sucrose doesn't get broken at very low temperatures. We can also try blanching potatoes before frying. This removes half the sugar, resulting in lower levels of acrylamide. In the future, safer potatoes may be available. The food industry is already being encouraged to use potato varieties that naturally produce less acrylamide. Growers are looking to develop varieties they contain less asparagines, an amino acid that seems to be important for making chemical.

\section{World Epidemic of Cancer}

The people can be poisoned as a victims of an unscrupulous food manufacturers, when the slice of bread, bowl of cereal or plate of waffles in front of them contain a potent cancer-causing chemical called acrylamide. The chemical might be impossible to eradicate, as it was created during the traditional cooking process. Acrylamide might be responsible for a long lasting and not enough known World Epidemic of Cancer. The toxic industrial chemical acrylamide is lurking in a huge range of World's everyday foods. The discovery meant that almost everyone must be ingesting this toxic substance, with unknown consequences for public health [1].

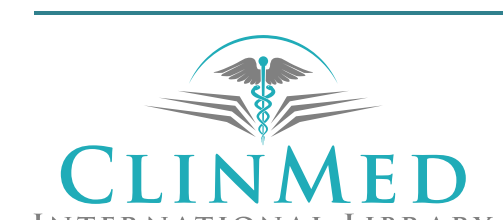

INTERNATIONAL LIBRARY
Citation: Skopec R (2017) Main Producers of World Cancer Epidemic. Int Arch Intern Med 1:003. Received: July 24, 2017: Accepted: November 25, 2017: Published: November 27, 2017

Copyright: (C) 2017 Skopec R. This is an open-access article distributed under the terms of the Creative Commons Attribution License, which permits unrestricted use, distribution, and reproduction in any medium, provided the original author and source are credited. 
A diet that regularly includes red meat increases the likelihood of developing eight serious diseases, as it find new research of the National Cancer Institute (NCl). This is an observational study, told the lead author Arash Etemadi, epidemiologist with the $\mathrm{NCl}$. The study found that the higher one's red meat consumption, the more the risk increased for developing cancer. The study has a 16-year follow-up, and the eating habits of more than half-a-million adults between the ages of 50 and 71. Compared with the one-fifth of people who ate the least of red meat, the one-fifth who ate the most had a 26 percent increased risk of death from various causes. The study looked at both unprocessed meat and those processed to include higher levels of nitrites and nitrates, already linked to numerous health risks [2].

This isn't the first study to link red meat consumption with health risks; other research has found red meat consumption is connected to a greater risk of developing high cholesterol and heart disease. I have also been linked to an increased risk of developing breast cancer as well as increased risk of obesity.

By contrast, a vegetarian diet has been linked to decreased risk of many of the same illnesses including heart disease, diabetes, and cancer. The American Medical Association (AMA) recently urged hospitals to reduce animal offerings and instead add more plant-based menu options to their cafeterias and patient meals, to improve the health of patients, staff and visitors. There is 8 serious diseases linked to red meat consumption, it is the conclusion of the National Cancer Institute study.

The $\mathrm{NCl}$ study comes just as California considers legislation known as AB 243, which would double the funding for the state's beef check off program aimed at amplifying up the USDA efforts to increase beef consumption, like the "Beef: It's What's for Dinner" promotions. Your red Meat Addiction is Killing More Than Just the Cow: Widespread Extinction Linked to Beef Production [3].

\section{Waist as the Risk Factor of Cirrhosis and Cancer}

It was several times demonstrated that a build-up of fat around the waist can cause more serious complications than obesity in the development of Non-Alcoholic Fatty Liver Disease (NAFLD). NAFLD is a condition in which fat builds up in the liver. Partly, this accumulation of fat can cause inflammation of the liver and eventually lead to permanent scarring (cirrhosis), which can seriously impair the liver's ability to function. NAFLD is a condition strongly linked to obesity, with prevalence as high as $80 \%$ in obese patients. The lean form of the disease can lead to diabetes, high blood pressure, cancer and even death. Similar problems have been reported only in $16 \%$ of individuals with a normal body weight.

As it said Dr. Rosa Lombardi from the University of Milan, their study for the first time show that patients with lean-NAFLD (not actually obese) who have increased levels of waist fat can in fact be at greater risk than obese patients with NAFLD. The researchers in the Italian study evaluated the features of lean-NAFLD in 323 patients with biopsy-proven NAFLD. Subjects were divided according to $\mathrm{BMI}\left(<25 \mathrm{~kg} / \mathrm{m}^{2}\right.$ defined as lean-NAFLD), waist circumference and abdominal fat.

The study found that NAFLD patients with a waist circumference greater than 35/40 inches, females/males, respectively, was significantly associated with metabolic syndrome $(p=0.001)$ (the combination of diabetes, high blood pressure and obesity), carotid plaques ( $p=$ 0.03 ) (the build-up of fatty substances and cholesterol deposits in the carotid artery) and significant fibrosis ( $p$ $=0.03$ ) (the first stages of liver scarring), compared to obese patients with NAFLD. This was true even in patients with normal weight (lean-NAFLD).

The research also suggested that metabolic, cardiovascular and tissue complications caused by NAFLD can be more effectively detected by combining Body Mass Index (BMI) and waist measurements.

Above study has proven to us that the severity of non-alcoholic fatty liver disease is not necessarily linked to how obese an individual is, but instead how much fat build-up they have around the waist. The results have highlighted the need for additional research into how analyzing someone's waist and not just their weight, is important in detecting individuals at risk for complications associated with above disease. Many diabetics don't even know they have serious liver disease [4-6].

\section{Platelet's $\alpha 6 \beta 1$ Integrin Participates in Tumor Metastasis}

Metastasis of cancer cells to sites distant from the primary tumor is the leading cause of cancer-related death. There is growing evidence that platelets aid the dissemination of cancer cells. In their study published by $\mathrm{JCl}$ Insight, Pierre Henri Mangin and colleagues at the Etablissement Francais du Sang-Alsace have shown that a molecule expressed on platelets, known as $\alpha 6 \beta 1$ integrin, participates in tumor metastasis. Doing so by promoting interactions between tumor cells and platelets [1].

Compared to control animals, mice lacking $\alpha 6 \beta 1$ integrin specifically on platelets exhibited decreased lung metastasis after injection of tumor cells intravenously or into the mammary fat pad. Mangin and colleagues determined that the tumor cell protein ADAM9 binds platelet $\alpha 6 \beta 1$ integrin to promote platelet activation and tumor cell extravasation.

Importantly, antibody-mediated blockade of $\alpha 6 \beta 1$ integrin inhibited tumor metastasis in murine models of breast cancer and melanoma. These finding suggests that disruption of tumor/platelet interactions could prevent metastasis [7].

There is growing appreciation that the current obe- 
sity epidemic is associated with increases in cancer incidence at a population level and may lead to poor cancer outcomes. The diagnosis and treatment of cancer may mask the obesity effects. An association of obesity with cancer is biologically plausible because adipose tissue is biologically active, secreting estrogens, adipokines and cytokines. In obesity, adipose tissue reprogramming may lead to insulin resistance, with or without diabetes and it may contribute to cancer growth and progression locally or through systemic effects.

Obesity-associated changes impact cancer in a complex fashion, potentially acting directly on cells through pathways, such as the Phosphoinositide 3-kinase (PI3K) and Janus Kinase-Signal Transducer and Activator of Transcription (JAK-STAT) pathways, or indirectly via changes in the tumor microenvironment. The potential for pharmacologic interventions can target the obesity cancer metastasis link [1].

\section{Withdraw of Cholesterol Caused Tumor Regres- sion}

Depriving deadly brain cancer cells of cholesterol, which they import from neighboring healthy cells, specifically kills tumor cells and caused tumor regression and prolonged survival in mouse models. Findings published in Cancer Cell show a potential method for treating Glio Blastomas (GBM), the most common and most aggressive form of brain cancer. GBMs are extremely difficult to treat. The median survival rate is just over 14 months, with few treated patients living five years or more past diagnosis.

Adult brain cancers are almost universally fatal, in part because of the biochemical composition of the Central Nervous System (CNS) and the blood-brain-barrier. This selectively and protectively limits the passage of molecules from the body into the brain, but which also blocks most existing chemotherapies, contributing to treatment failure.

That includes blocking small molecule inhibitors that target growth factor receptors, which have not proven to be effective with brain cancers, probably due to their inability to get past the blood-brain barrier and achieve sufficiently high levels in the CNS. Drugs designed to target these oncogenes have difficulty accessing their targets in the brain.

The oncogenes as mutated genes can rewire the biochemical pathways of cells in ways that make them dependent on proteins that are not themselves encoded by oncogenes. Targeting these oncogene-induced co-dependencies opens up a much broader pharmacopeia; including the use of drugs that aren't traditionally part of cancer drug pipelines but have better pharmacological properties.

Interestingly, GBM cells cannot synthesize cholesterol, which is vital to cell structure and function, particu- larly in the brain. Instead, GBM cells derive what they need from brain cells astrocytes, which produce cholesterol in abundance. Roughly 20 percent of total body cholesterol is found in the brain.

When normal cells have sufficient cholesterol, they convert some of it into molecules oxysterols, which activate a receptor in the cell's nucleus - the Liver X Receptor (LXR) - to shut down the uptake of cholesterol. When normal cells get enough cholesterol, they stop making it; stop taking it up and start pumping it out. In GBM cells, this mechanism is completely disrupted.

GBM cells ensure their cholesterol supply by suppressing the production of oxysterols, ensuring cell's LXRs remain inactive.

Disrupting cholesterol import by GBM cells caused dramatic cancer cell death and shrank tumors significantly, prolonging the survival of the mice! The strategy worked with every single GBM tumor and even on other tumors that had metastasized to the brain. LXR-623 also had minimal effect on astrocytes to other tissues of the body [8].

\section{High Fat Diets Alter Gut Bacteria}

Researchers have shown a high fat lead to specific changes in gut bacteria which can fight harmful inflammation as precursor of cancer and Crohn's disease. In the new study, a diet of plant-derived "good" fats, including coconut oil or cocoa butter, drastically reduced bacterial diversity in mice with above diseases. Mice fed beneficial fatty diets had up to thirty percent fewer kinds of gut bacteria as those fed a normal diet, collectively resulting in a very different gut microbial composition. Some of the species changes showed up in feces, while others were different in caecum, a portion of the intestine commonly inflamed. Mice fed even low concentrations of coconut oil or cocoa butter also had less severe small intestine inflammation.

The finding is remarkable because it means that patients could also have a beneficial effect on their gut bacteria and inflammation by only switching the type of fat in their diet. As it told Professor Alexander Rodriguez-Palacios, first author of the above study, patients would only need to replace a "bad" fat, and eat normal amounts.

The study is one of the first to identify specific changes in gut bacteria (our microbiome) associated with disease. It is also show how high fat diets can alter gut bacteria to combat inflammation. Results from the study could help doctors identify bacteria to use in probiotics to treat patients suffering from inflammatory bowel syndromes. Ongoing studies are now helping us to understand which component of the good and bad fats make the difference in the gut microbes and make mice healthier. To identify the good fat-loving microbes help testing their selection for probiotics. 
These finding may have varying effects for patients. Not all good fats must be good in all patients. Mice indicate that each person could respond differently. Diet is something we are very hopeful could help at least some patients without side-effects and risks carried by drugs. Now is the time to really discover what makes fat good or bad for inflammation and i.e. cancer [9].

\section{Molecular Mechanisms of the Anti-Cancer Effect of Nuts}

Walnuts can inhibit the growth of cancer cells. Nutritionists at University Jena present results from a recent study, which throw light on molecular mechanisms of this protective effect. Roasted and salted, ground as a baking ingredient or fresh from the shell, for all those who enjoy eating nuts, there is good news. Their latest research shows that nuts can inhibit the growth of cancer cells.

For a long time now we have known that nuts are full of substances that are good for the heart and the cardiovascular system, or that protect against becoming overweight or developing diabetes. Some studies have also indicated a protective effect against colon cancer. What we have not known in detail up to now is what this effect of nuts is based on. In a publication in the specialist journal Molecular Carcinogenesis, they present results which throw light on the molecular mechanisms of this protective effect.

According to this study, nuts have a positive effect on health because they are involved in activating the body's own defences for detoxifying reactive oxygen species. Such substances, which are created by ultraviolet radiation, various chemicals, or distinct food metabolites, for example, can cause DNA damage that leads to cancer development. The body has a whole series of protective mechanisms that render reactive oxygen species harmless. The nutritionists in Jena now shown that these mechanisms are stimulated by nuts and the substances they contain.

The researchers investigated the effect of five different types of nuts: Macadamia nuts, hazelnuts and walnuts, as well as almonds and pistachios. The nuts were artificially digested in test tubes and the effects of the resulting digestion products on cell lines were then analyzed. The researchers established that the activity of the protective enzymes catalase and superoxide dismutase increases in the cells that are treated. The digestion products induce what is called programmed cell death in the cancer cells thus treated.

This effect is mediated by all the types of nuts studied, noted Prof. Michael Glei, who lead the study. In the next stage, they want to find out whether this protective effect is reduced by roasting the nuts. As most of the nuts investigated are predominantly consumed in roasted form, this further research may enable to give appropriate nutritional advice based on the results. A handful of nuts a day cut the risk of a wide range of diseases [10].

\section{Gold Nanoparticles Block the Function of Cancer Cells}

The main factor involved in fatal cancer is metastasis, when cancer spreads from the original tumor to different parts of the body. A new study claims to found a way to stop cancer cells from migrating to other parts, a key process in metastasis. The team believes their discovery could lead a treatment that could slow down this process or even stop it all together.

The researchers were able to use nanotechnology to create extremely small materials that prevent cancer cells from moving, i.e. breaking cancer legs. If the cancer can't move, it can't metastasize. If cancer stays in a tumor in one place, you can get to it and it's not so likely to kill the patient. When it spreads around the body, that's what really makes it deadly, said lead researcher Mostafa El-Sayed.

The method appears to be very effective as a locally administered treatment that also protects the body from cancer's spread away from the treated tumors and it is also very mild, it can be applied many times over if needed. Cells move using leg-like protrusions called filopodia, but cancer cells produce far more of these than healthy tissue. While cancer cells that try to spread usually die at some point in the process, if conditions are favorable, the cancer can successfully form a tumor in another part of the body.

The team was able to obstruct the movement of these filopodia using gold nanoparticles specifically designed so that they only block the function of cancer cells and do not interfere with healthy cells. They found that shining a low-energy laser at the cells caused the cell movement to stop completely. The laser caused that the gold heat up and partially melts the cancer cells legs, completely stopping their movement.

The gentle laser didn't burn the skin or damage tissue, so it could be dosed multiple times and more thoroughly stop the cancer cells from being able to travel. According to the National Cancer Institute, metastasis is the main reason that cancer is so deadly. The metastatic cancer cells can remain inactive on a site for many years before they begin to grow again.

When the cells move to other nearby tissue, it can be very difficult to control. This type of cancer is largely untreatable. Most treatment for metastasized cancer aims to stop or slow down its spread. Some of the most common sites of metastasis are the bone, liver and lung.

Although the treatment is still in the research phase, the team sees it being used for head, neck, breast and skin cancers with direct local injections. They also suspect they would be able to treat deeper cancer using a fiber optic or endoscopic laser [11]. 
Agriculture as Producer of World Cancer Epidemic

If there is a trouble with growing fruits and veggies in the garden because of weeds, insects, or small animals, probably will be used soil containing pesticides to help produce grow. Pesticides ward away the living things that can sabotage the summer crops, but they are also carcinogenic. A growing number of studies are finding evidence that pesticides used in home gardening and agriculture are associated with increased cancer risk.

The exact ingredients in the pesticides that can cause cancer are discussed, though some chemicals, such as glyphosate, were detected frequently. While humans are choosing to use pesticides near home, then wear preventative to ensure the pesticides don't touch the skin.

Glyphosate-based herbicides are leading products of the current pesticide market, and this trend has further strengtened outside Europe with the spread of glyphosate tolerant GM crops. Glyphosate forms complexes with metal ions ( $\mathrm{Al}, \mathrm{Fe}, \mathrm{Mn}, \mathrm{Zn}$ ) in the soil, but precipitation washes it into deeper soil layers. Its primary metabolite, AMPA, is more mobil than the parent compound. Their detection in environmental samples is rather problematic, this is why these compounds are not listed in most countries among commonly detected pesticide residues.

The expansion of glyphosate-tolerant GM crops is likely to result in more common occurrence of glyphosate in food and feed, and metabolites AMPA and NAG may also call for special attention depending on given genetic events.

Severe professional debates are going on regarding the side-effects of glyphosate and its herbicide preparations (particularly those containing POEA as formulating agent). Glyphosate reduces disease tolerance of certain plants, while facilitating fungal root colonization e.g., Fusarium species. It is toxic to amphibians, and is suspected to exert teratogenicity on birds and amphibians.

Mutagenicity and carcinogenicity of glyphosate-based preparations are proved in debates. The hormone modulant effect of glyphosate is exacerbated by the formulating agent (POEA). Upon extended application, Glyphosate Resistant (GR) populations are selected from given weeds: The number of such species identified is 21 , including GR Amaranthus, Conyza and Lolium populations besides ragweed (Ambrosia artemisiifolia) and Johnsongrass (Sorghum halepense) [12].

\section{The Food Supply through Agricultural Use}

Some compounds find their way into the food supply through agricultural use, animal farming, or food processing, even if their use is not directly intended for human consumption. Examples include growth hormones or antibiotics used in animal farming, some amounts of pesticides and herbicides in plant-based foods, and compounds like as Bisphenol A (BPA) or phthalates that enter food from packaging. Several of these compounds are not known to directly cause cancer, but they may influence cancer risk in other ways - for example, by acting as hormone like substances in the body.

Unintended contamination of food may result in exposure to chemicals that are a cause of concern and may be related to cancer risk. Examples include heavy metals such as cadmium or mercury. These metals may enter the food supply if they build up the food chain, such as from fish, or they may enter through contamination or their natural presence in soil or water.

For many other compounds for which the effects on cancer risk are not clear, there may be other good reasons to limit exposure. Food processing may also alter foods in ways that might affect cancer risk. An example is the refining of grains, which greatly lowers the amount of fiber and other compounds that may reduce cancer risk.

The processing of meat, by adding preservatives such as salt or sodium nitrite to prevent the growth of germs or smoking the meat to preserve or enhance color and flavor, may add compounds that might increase the potential of those foods to cause cancer. Studies have linked eating large amounts of processed meats with an increased risk of colorectal cancer. This may be due to nitrites, which are added to many lunch meats, hams, hot dogs and other processed meats.

Irradiation of food products is one way to limit the risk of germ contamination and food poisoning. Irradiated meats and other foods are also widely available. Because radiation is known to cause cancer, there has been concern that food irradiation may present cancer risk. Radiation does not remain in foods that have been irradiated [13].

\section{Role of Food Additives in Cancer Risk}

Concern about the possible effects of food additives on health, including cancer, is one reason that many people are now interested in organic foods. Organic foods are often promoted as an alternative to foods grown with conventional methods that use chemical pesticides and herbicides, hormones, or antibiotics. These compounds cannot be used for foods labeled as "organic". Organic foods, as defined by the US Department of Agriculture (USDA), also exclude genetically modified foods or foods that have been irradiated. Vegetables, fruits and whole grains should form the central part of person's diet, regardless of whether they are grown conventionally or organically.

Many substances are added to foods to prolong shelf and storage life and to enhance color, flavor, and texture. The possible role of food additives in cancer risk is an area of great public interest. 
While not a household product itself, food dye is found in nearly processed food, drink, vitamin, and dietary supplement found in human consumption. Studies have shown 9 types used in the U.S. could be carcinogenic. Use of these dyes has increased fivehold in the U.S. since 1955 and the Center for Science in the Public Interest [13] published a summary on what they found when testing various food dyes. It's first important to note the studies were performed on rodents and lasted for two years, so we yet to understand exactly how humans could be affected over a long period of time.

The cited report found Blue 2, Citrus Red 2 and Yellow 6 all caused tumors in the rodents. The CSPI believes that these dyes have no place in humans food. Citrus Red 2 appeared to be safe when used to make orange peels brighter, for example, but could be more risk when ingested. More testing is certainly needed to prove the mechanism with which dyes affect humans $[13,14]$.

\section{Conclusions}

It is well known that processed meats - such as bacon, sausages and ham - do cause cancer, according to the World Health Organization (WHO). Its report said 50 $\mathrm{g}$ of processed meat a day - less than two slices of bacon - increased the chance of developing colorectal cancer by $18 \%$. It also said red meats were probably carcinogenic too. These all could be increasing the risk of cancer. High temperature cooking, such as on a barbeque, also create carcinogenic chemicals. The WHO has come to these conclusions on the results of its International Agency for Research on Cancer, which assesses the best available scientific evidence.

It has now placed processed meat in the same category as plutonium, but also alcohol as they definitely do cause cancer. For an individual, the risk of developing colorectal (bowel) cancer because of their consumption of processed meat remains this risk increases with the amount of meat consumed, Dr. Kurt Straif from the WHO said. Estimates suggests 34,000 deaths from cancer every year could be down to diets high in processed meat.

That is in contrast to one million deaths from cancer caused by smoking and 600,000 attributed to alcohol each year. The WHO said there was evidence that 100 $\mathrm{g}$ of red meat a day increased the risk of cancer by $17 \%$. The also said its findings were important for helping countries give balanced dietary advice.

In above our article we are outgoing from several sources published in scientific journals. On this base we are coming to conclusions that:

- For long times humans are suffering under a dangerous World Epidemic of Cancer,

- From this reason all Human Civilization must make general corrections in its Eating Habits,
- First of all, humans must gradually stop to eat the processed meat and the red meat in its own best interests,

- The until today traditional methods of preparing foods for human consumption we must stop to use next methods of cooking as minimum: Cooking at high temperatures, cooking over $120^{\circ} \mathrm{C}$, baking, toasting, frying, and roasting starchy foods, etc.

- As it was published by Marianne Thyssen from European Union (EU), better protection against cancer-causing chemicals could save 100,000 lives. EU rules to limit to 13 cancer-causing chemicals at the workplace could save these amount of lives in the next 50 years. The Commission is proposing changes to the EU's Carcinogens and Mutagen Directive, specifically new or amended limit values which set maximum concentrations for the presence of chemical carcinogen in the work place air.

The number of deaths attributed to occupational cancer in the EU is reported to be around 102,000 per year. According to the WHO, cancer is the second largest cause of death in most developed countries, but in the European Union cancer is the first cause of deaths. $53 \%$ of work-related deaths is due to cancer, compared to $28 \%$ for circulatory diseases and $6 \%$ for respiratory diseases.

We must say this openly, that consumption of processed meat and red meat, due the cancer-causing chemicals like acrylamide, are poisoning modern age people, making them patients and deaths. These carcinogenous eating habits are causing enormous economic and financial deficits to Western countries economies. The explanation of these problems may give on deeper level of Hard Science. The World is coded by the Quantum Entanglement Entropy (QEE), so we think that this a "God's Code". The QEE means a selection, much more complex than Darwinian one, through the coding by Quantum Entanglement Entropy, leading to consciousness explained without the errors of over-simulations of the science ruling before the Quantum Physics Era [15].

Agriculture is today the main producer of the World Epidemic of Cancer! Pesticides ward away the living things that can sabotage the summer crops, but they are also carcinogenic. A growing number of studies are finding evidence that pesticides used in home gardening and agriculture are associated with increased cancer risk.

The exact ingredients in the pesticides that can cause cancer are discussed, though some chemicals, such as glyphosate, were detected frequently. While humans are choosing to use pesticides near home, then wear preventative to ensure the pesticides don't touch the skin. 
The expansion of glyphosate-tolerant GM crops is likely to result in more common occurrence of glyphosate in food and feed, and metabolites AMPA and NAG may also call for special attention depending on given genetic events.

By contrast, a vegetarian diet has been linked to decreased risk of many of the same ilnesses, first of all including cancer. The American Medical Association recently urged hospitals to reduce animal offerings and instead add more plant based menu options to their cafeterias and patients meals, to improve the health of patients, staff and visitors.

\section{References}

1. Goodwin PJ, Stambolic V (2015) Impact of the Obesity Epidemic on Cancer. Annual Review of Medicine 66: 281-296.

2. Coleman MP, Estève J, Damiecki $P$, Arslan A, Renard H (1993) Trends in cancer incidence and mortality. IARC Sci Publ 121: 1-806.

3. Hertz DL, Rae J (2015) Pharmacogenetics of Cancer Drugs. Annual Review of Medicine 66: 65-81.

4. Anna Christina Dela Cruz, Elisabetta Bugianesi, Jacob George, Christopher P Day, Hammad Liaquat, et al. (2014) 379 Characteristics and Long-Term Prognosis of Lean Patients With Nonalcoholic Fatty Liver Disease. Gastroenterology 146: S-909.

5. Milic S, Lulić D, Štimac D (2014) Non-alcoholic fatty liver disease and obesity: Biochemical, metabolic and clinical presentations. World J Gastroenterol 20: 9330-9337.
6. Ren-Nan Feng, Shan-Shan Du, Cheng Wang, Yan-Chuan $\mathrm{Li}$, Li-Yan Liu, et al. (2014) Lean-non-alcoholic fatty liver disease increases risk for metabolic disorders in a normal weight Chinese population. World J Gastroenterol 20: 17932-17940.

7. https://www.news-medical.net/news/20160909/Interactions-between-tumor-cells-and-platelets-could-lead-to-cancer-metastasis.aspx

8. Mischel (2011) Targeting cholesterol to fight deadly brain cancers. Cancer Cell.

9. https://www.cheatsheet.com/health-fitness/cancer-from-chemicals-household-products-that-are-known-carcinogens.html/3/

10. Wiebke Schlörmann, Julia Lamberty, Stefan Lorkowski, Diana Ludwig, Henning Mothes, et al. (2017) Chemopreventive potential of invitro fermented nuts in LT97 colon adenoma and primary epithelial colon cells. Molecular Carcinogenesis 56: 1461-1471.

11. Ali MRK, Wu Y, Tang Y, Xiao H, Chen K, et al. (2017) Targeting cancer cell integrins using gold nanorods in photothermal therapy inhibits migration through affecting cytosceletal proteins. Proc Natl Acad Sci U S A 114: E5655-E5663.

12. Darvas B (2011) Environmental Health Problems of Glyphosate Applications. Nǒvényvédelem 47: 401.

13. (2017) The Center for Science in the Public Interest (CSPI).

14. The American Cancer Society (ACS) medical and editorial content team (2016) Food additives, safety, and organic foods.

15. Skopec R (2017) Coding by Quantum Entanglement Entropy. NeuroQuantology 15: 200-207. 